after preliminary treatment to improve the general health and to combat infection.

\section{BIBLIOGRAPHY}

HOMANS, J. (1939), 'Circulatory Diseases of the Extremities.' The Macmillan Co. New York.
LEARMONTH, J. R., BLACKWOOD, W., and RICHARDS, R. L. (1944), Edin. Med. Fourn., 51, I.

LEWIS, Thomas (1936), 'Vascular Disorders of the Limbs,' Macmillan and Co. London.

Medical Research Council Memorandum (1944), No. 13, 'Arterial Injuries.'

PRIESTLY, J. B. (1932), Fourn. Nerve and Mental Dis., 75, 137. RICHARDS, R. L. (1946), 'The Peripheral Circulation in Health and Disease,' $E$. and S. Livingstone (Edin.).

\title{
OBSERVATIONS ON THE ORGANIZATION OF CARDIOLOGY UNDER THE NATIONAL HEALTH SERVICE ACT
}

\author{
By Edmund Finsterbush, M.D.(Vienna), M.R.C.S.(Eng.), L.R.C.P.(London) \\ Consulting Cardiologist to the M:nistry of Pensions
}

It may be assumed that the organization of provincial hospitals will present ma y a difficult problem during the transitional stage of the inception of the National Health Service Act, 1946, owing to the great differences in organization, staffing, and clinical standards in municipal hospitals on the one hand and in voluntary hospitals on the other, as well as to the spirit of competition and isolation prevailing among institutions and doctors alike. Moreover, subjects such as psychiatry, dermatology, cardiology, etc. are novelties to a provincial hospital and will, as such, further aggravate the position. It may therefore not be out of place to outline some basic ideas of organization of a cardiological service.

\section{Pathology}

... for the signs of the dead body furnish invaluable data from which it is desirable sometimes to explain diagnosis, sometimes to justify, and always to learn.' (Sir George Newman, ' Notes on Medical Education' I9I8.)

There can be no real application of up-to-date clinical science without close and wholehearted co-operation of competent pathologists and radiologists with the physician. In the first place, post-mortem dissections must be performed as a routine, since they are undoubtedly the foremost tutor, highest examiner and corrector of the physician. Pathology is the sub-structure of diagnosis. (W. Boyd.) This may sound commonplace, yet must be constantly emphasized, for I am convinced that the lack of expert dissections in provincial hospitals is the main cause of the low clinical standard which prevails outside the teaching centres.
A few figures will clinch my statement. In a municipal borough somewhere in England in 1935 the total number of deaths was 1,368 ; of these 529 , (i.e. 38.6 per cent.) resulted from cardiovascular disease. In would-be compliance with the regulations made by the Registrar-General, the immediate cause of death in this group of 529 was certified as myocarditis in 25 , as syncope in 31 , and as cardiac dilatation in 21 cases. Arteriosclerosis as the sole cause of death occurred seven times. The extent of post-mortem studies in this group can be seen by the following. Out of the total of 529 deaths from cardiovascular disease 142 occurred in municipal hospitals, and $4 \mathrm{I}$ in the voluntary hospital. Among the former, two dissections took place, including one on the coroner's order; among the latter, seven dissections were performed spontaneously and one on the coroner's authority. In 1946 , the position in the same borough was that out of a total number of 1,526 deaths, 669 , (i.e. 43.8 per cent.), were ascribed to cardiovascular disease, among which 182 died in municipal hospitals, and 45 in the voluntary hospital. In the former, six post-mortem dissections were performed, including one on the coroner's order; in the latter twelve dissections were obtained on the hospital's own initiative, and six on the coroner's order. In this group of 669 deaths arteriosclerosis was certified as the immediate and sole cause 29 times ; myocarditis, both acute and chronic, was certified in 53 cases (sic), most ranging between 60 and 89 years of age. Some illustrative post-mortem diagnoses of 1946 may be quoted.

M.63 ' Cerebral haemorrhage due to toxaemia 
and failing vascular system.' (Coroner's post-mortem in general practice.)

M.77 'Coronary embolism. Arteriosclerosis.' (Coroner's post-mortem in general practice.)

F.59 'Cerebral haemorrhage.' (Coroner's post-mortem in hospital.)

M.57 'Haemoptysis from lung infarction due to arteriosclerosis.' (Coroner's postmortem in general practice.)

F.46 'Portal thrombosis.' (Post-mortem in hospital.)

M.73 'Angina due to atheromatous valves and coronary arteries.' (Coroner's postmortem in general practice.)

The significance of a correct clinical diagnosis as an essential prerequisite of remedial and preventive medicine has been emphasized by leading authorities for some considerable time. The fundamental importance of correct diagnosis, both clinical and post-mortem, has recently been stressed by Prof. J. Ryle (1946): 'The social pathology of a disease is largely dependent upon reliable clinical, statistical, and social data, and especially upon morbidity and mortality figures.'

The technique of autopsy of cardiovascular material must comprise detailed weighing and measuring of the heart as a whole, of its individual chambers, and of its valvular orifices respectively ; also probing of the coronary artery tree, and histological studies whenever they may be required. Only by diligent search of the patient's environmental background, by systematic recording of the course of disease, and by documentation of post-mortem data and persistent correlation of the latter with the symptoms and clinical findings in individual cases can we deepen our understanding and knowledge of disease. 'Those who have dissected or inspected many bodies have at least learned to doubt; when others, who are ignorant of anatomy and do not take the trouble to attend to it, are in no doubt at all.' (Morgagni quoted by W. Boyd.)

\section{Team Work}

In the second place, team work of the whole staff in general, and co-operation with the radiologist and clinical pathologist in particular, must be promoted by all possible means. The pathologist must be looked upon as the main pillar of a modern hospital. Prof. J. A. Nixon has recently quoted an instance of one of the most shocking of the workhouse infirmaries which raised itself into an efficient Emergency Medical Service hospital largely as a result of having a first-class pathologist. ('The Hospital Service of the Future.' Royal Soc. Med. March 6, 1948.)

It is undesirable to exhibit drugs like the sul- phonamides, thiouracil, thiocyanates, dicumarol, etc., without careful frequent observation of the drug concentration in the blood, and without frequent haematological observations. 'It is imperative that total and differential blood counts be made at least twice weekly in patients still under sulphonamide treatment after the seventh day.' ( 'The Medical Use of Sulphonamides.' Med. Res. Council. I945. p.22). Diagnosis and treatment of rheumatic fever and bacterial endocarditis call for serial haematological and bacteriological observations during the early, sometimes obscure stages, throughout the course of overt malady, and during convalescence. Incipient changes of retinal vessels, a low-grade oedema of the optic disc in the course of arterial hypertensive disease call for expert ophthalmological opinion. The diagnosis and rat onal management of arterial hypertension may sometimes depend on the radiologist for urography to establish the underlying renal pathology, or on the neuro-surgeon for trans-diaphragmatic splanchnic nerve section with dorsolumbar sympathetic ganglionectomy, to name but a few examples. The same applies to peripheral vascular disease. Congenital heart lesions such as patent ductus arteriosus, pulmonary stenosis (Fallot's tetralogy), aortic coarctation or double aortic arch with obstruction of trachea should be referred to a thoracic surgeon well conversant with the latest advances in cardiovascular surgeryo whenever this is clearly indicated.

The recent triumphant advances of American surgery, e.g. pneumonectomy, resection of twothirds of the oesophagus, operations on some of the congenital heart lesions, are being ascribed rather to pre-operative preparation of the patient, to perfection of anaesthesia, and to good post-operative management rather than to mere perfection of surgical technique per se. What does pre-operative preparation imply ? The answer is : ancillary studies such as estimation of haemoglobin, haematocrit, blood-plasma proteins, $\mathrm{Rh}$-factor, crossmatching, etc. In other words, these ancillary investigations occupy the first place in modern surgery; they are indeed the alpha and omega of up-to-date clinical medicine as well. Nevertheless it must be remembered that no instrumental and laboratory studies however invaluable can ever replace intelligent comprehension of the patient's whole personality both physical and mental, together with careful evaluation of environmental factors as these may act upon an individual and his disease. No mere addition of the multitudinous analytical data but synthetical perception of the human organism, its total function, and behaviour, should be our aim. 'Diseases are abnormal, though not altogether irregular, behaviors of man.' (Sir Clifford Allbutt, 1906, 
quoted by Clendening and Hashinger. 'Methods of Diagnosis.' Washington 1947.)

At this point the question arises whather the type of team work outlined above can easily be attained in a provincial hospital at an early stage of the new Health Service. Pondering on the problem for some time, and knowing the conditions prevailing in the provincial hospitals one is forced to the conclusion that the problem can be solved only by the active and generous aid of the teaching or University hospital of the region concerned. Not only in cases of exceptional complexity or rarity is the teaching hospital's advice desirable, but its systematic guidance and criticism are strongly recommended.

\section{Organization}

The cardiological service should comprise from the very start a ward for in-patients to accommodate anything between ro and 20 cases, and a clinic for out-patients; adjoining the ward an appropriate room should be provided for complete examination of new cases. The timehonoured screening is no guarantee of privacy and quiet, both essential to gain the patient's confidence during the first interview. Albeit the point may seem to be a purely administrative one and not directly relevant, I should like strongly to emphasize the senseless torture of the sick caused by 'blaring' radios, a habit which has gradually crept into our hospitals. From early morning till late evening it is not unusual for the wireless to be on in a general ward, to the enjoyment of the convalescent and of the staff, perhaps, but to the detriment of those afflicted by fever, pain, or prostration. Only the individual who is familiar with the sensation of profound exhaustion or with the anguish of a recent fracture, pent-up pus, or myocardial ischacmia, can comprehend the cruelly aggravating effect of protracted noise. For the average cardiac case rest and quiet are the foremost therapeutics. "The watchword throughout treatment of congestion is indeed rest; rest for the body ; 'rest for the mind ; rest for the heart.' (Sir Thomas Lewis.) 'With absolute rest ... the stimulating effect of noises is reduced to a minimum.' (P. D. White.)

The clinic should be equipped with a few cubicles for the undressing of patients in privacy. Both the cardiac ward and clinic must be situated on the ground floor, otherwise a lift is indispensable for the transport of ambulatory and stretcher cases alike. It should be borne in mind that an in-patient does not remain absolutely stationary during his stay in hospital; he may have to be moved several times to the X-ray room, and during early convalescence he will benefit by graded exercises in the open. The conveyance of patients by means of stretchers over staircases in a hospital building devoid of lifts puts a great strain on the personnel and will ultimately become a serious handicap to the management of the hospital.

\section{Clinical Routine}

Haphazard methods of work are out of place in a well organized hospital. A daily round of the physician in charge is a matter of course, and so is the commencement of duty at 9 a.m. There must be time for observation at $t h \geqslant$ bedside, for serial recording of blood pressure, taking of cardiograms, X-ray screening, therapeutic measures, attendance at post-mortem dissections, consultations with other departments, and last but not least for writing up of case notes. If the latter are to be done efficiently a stenographer must accompany the physician throughout the round along with the nurse. Observations on clinical progress should be dictated to the shorthand-typist during the round in a lowered voice, the instructions on treatment, dietary, special investigations and tests, etc., should be given to the sister. The other very important medical auxiliary will be the electrocardiographer. At present there are hardly any well trained cardiographers outside the teaching hospitals. Steps should be taken now for their training by those cardiologists willing to undertake it. For the sake of economy the secretary could be employed as electrocardiographer as well, especially in the early stages of the new service. Relieved of the burden of secretarial and purely technical work the physician will find more time for observation of the sick in the ward. 'Clinical science is essentially observational.' (J. Ryle, 1931.)

The system of weekly clinics is outdated, and has no place in a statutory health organization on a nation-wide basis. To touch just the fringe of the abundant cardiological material, three sessions per week seem to represent the minimum requirement. Apart from the large-scale influx of new outpatients as from July 5 a steady increase of old cases from the clinic and ward attending for aftercare or follow-up is to be expected. As to morbidity figures may I quote my observation of a group of 425 unselected cases between 1943 and 1945, higher ages being prevalent. There were 76 cases of cardiovascular disease (i.e. 17.88 per cent.). Classified from the standpoint of the aetiological factor these included: Congenital lesions, I ; rheumatic fever, acute and chronic, 4 ; syphilis, I ; thyroid disease, 4 ; hypertension, 29 ; pulmonary hypertension (chronic cor pulmonale) 2; atheroma and sclerosis (aorta, coronary, cerebral), 33 ; cardiac neurosis, 2 : bacterial infection, 0 . The high incidence of cardiovascular disease is evident from the mortality figures mentioned on page 83, obtained in an English borough. 
They were 38.6 per cent. in 1935 , and 43.8 per cent. in 1946 .

Quality before quantity should be our aim. This means a limited number of cases during a session, and therefore an evenly spaced appointment system in the out-patient department. It should be noted that the routine cardiological examination consists of four stages: history, clinical examination, radioscopy and electrocardiography. A laboratory test may also be needed in addition on occasions. Room should be left for emergency cases interrupting the routine work, e.g. status anginosus, or paroxysmal tachycardia. The ambulatory case requiring urgent admission must be spotted at once, for it is unwise to send home a specific aortitis who may expire next day. A rigid appointment system is being urgently recommended for the sake of the patients as well. Long waiting hours in cold draughty halls or corridors are a source of discontent and aggravate the patient's psychological state. Again and again I have had to listen to the patient's sad story of trepidation and distressing anxiety caused by the prolonged waiting in the out-patient department.

Overwhelming numbers will be detrimental to the success of the new Health Service. In the first place, the physician must have ample time to maintain the highest possible standard of work. In the second place, the few clinics and wards established, in the provinces of course, should be gradually developed to a pattern upon which the organization of hospital services may be based in future. In the third place, in order to reconcile the public and medical profession to the novel idea, and to rouse their interest and wholehearted co-operation during the early stages of the National Health Service it is imperative to offer them a small but perfect skeleton rather than a mammoth bulk of perfunctory and bungling machinery. On humanitarian grounds, I look for development of hospitals within the orbit of the National Health Service Act, 1946, as a first-rate priority. 'Unfortunately, first-class hospitals are in a very considerable minority.' (L. A. Parry, Lancet, December I3, 1947.) 'Many of these old people can only be treated satisfactorily in hospital ... but in the meantime the wretchedness of many decrepit old folks, dragging out a miserable existence under conditions that can commonly be described as shocking, is unworthy of a civilized society.' (P. Ferguson, Lancet, March I3, 1948.) It will be a source of inspiration to regional health authorities to remember that according to civil estimates for the year ending March 3I, I949, $f_{102,410,000}$ will be made available for hospital, specialist and ancillary services in England alone, compared with $£ 330,775$ in the year ending March 31, 1948. This three hundred fold increase will certainly impart more freedom of action to the enthusiastic organizer.

\section{Contributions of Local Authorities}

Owing to the prevailing conditions there is no prospect of early erection of an adequate number of modern hospitals; local authorities should, therefore, be encouraged to modernize, equip, and enlarge their workhouses, infirmaries, and hospitals. Here and there, a few bathrooms, a few cubicles, a new laboratory, a post-mortem room, a lift, a new storey as superstructure, etc., may all make a great difference.

\section{Nursing Personnel}

The physician in charge or medical officer must keep an eye on nurses' work, e.g. administration of injections, strict adherence to patient's dietary, times of medication, etc. I happen to have come across one medical ward in which hypodermic, intramuscular, and intravenous injections alike were administered by means of a fine short hypodermic needle in a slowly boring fashion, with deliberation and hesitation instead of by a single well determined puncture. Following this the piston was depressed with irritating slowness, and so the torture was completed. All this may appear to be mere pedantry, yet to the patient it is 8 source of unnecessary suffering, and can easily be avoided.

\section{Filing System}

This should be adopted on the approved pattern of one of the teaching hospitals.

\section{Physician's Time Table}

The rule must be laid down that the physician's work is to commence at 9 a.m., if he is to accomplish the various duties of a morning such as ward rounds, out-patients, examination of new cases admitted, attendance at autopsies, personal consultation with ancillary workers, etc. Assuming that the physician's work is arranged on a sessional basis, viz. three to five mornings weekly, this by no means implies his being off duty during the afternoon. Indeed, it stands to reason that a morning's clinical work means in turn many hours in the afternoon of preparing notes and reports, interprsting cardiograms, looking up references relevant to a clinical problem in hand, etc. This secretarial part of the work may, of course, be performed at the physician's home. Apart from this it must be borne in mind that he will have to devote a considerable part of his working day to reading. Extensive study of the literature on his own subject, as well as on general medicine, is a sine qua non be he engaged merely in clinical routine, and/or in research and teaching. Those 
endowed with the capacity and inclination for research work should be afforded every opportunity to pursue their course.

With the scope and variety of work indicated above there will be little time left for recreation of mind and body. Therefore, it would appear that four full morning sessions will in fact absorb the best part of the physician's working week. To maintain his fitness, enthusiasm, courage, cheerfulness and amiability he will have to repose on Nature's bosom two days a week, apart from an adequate yearly holiday. Only through generous reward and guarantee of social security can efficiency of the service be maintained, and ever more competent and suitable men attracted.

\section{ADDENDUM}

I am grateful to Prof. J. A. Ryle for encouragement to publish, and to Dr. Maurice Davidson for his unfailing generosity, and his helpful criticism of this paper.

TABLE

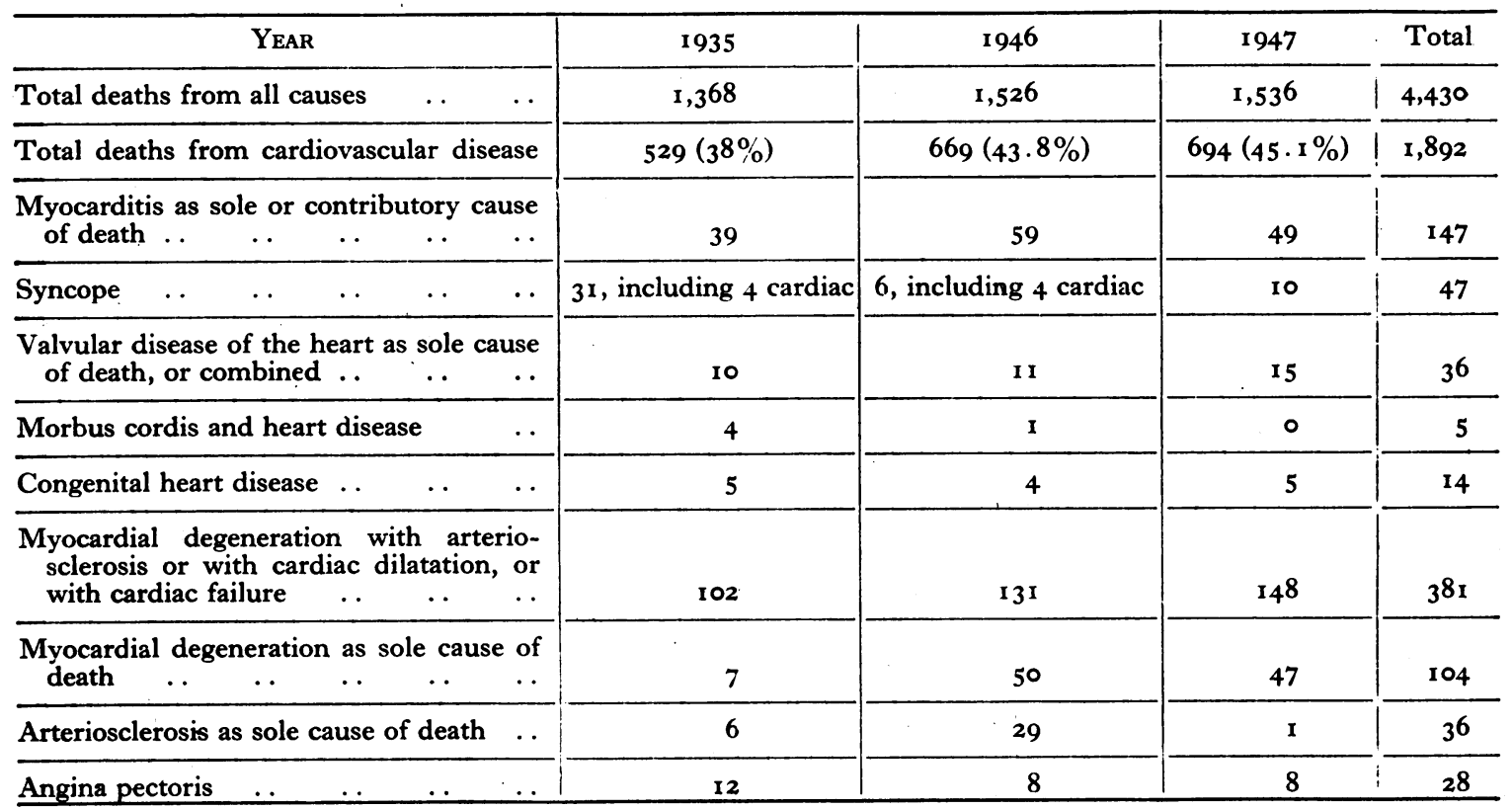

Deaths in an English municipal borough.

\section{BOOK REVIEWS}

\section{INTRODUGTION TO DISEASES OF THE CHEST}

By James Maxwell, M.D., F.R.C.P. 3rd Edition. Pp. xi +307 , with 66 illustrations. London : Hodder and Stoughton. 1948. I2s. 6 d.

This book, now in its third edition, will be well known to many readers. The basic facts affecting the diagnosis and treatment of the more common chest diseases are clearly and concisely set out. The chapters on the symptoms and physical signs are exceptionally plain. As the author points out, radiological investigation of chest disease is essential for early diagnosis, and more space has therefore been given to the description and interpretation of radiographic appearances. The 60 radiographs which illustrate the text are excellently reproduced so that the reader can really see the essential features. Treatment with penicillin and the various sulphonamide drugs is adequately discussed. The value and method of use of streptomycin are rightly considered to be too ill-defined to be included at present.

In a short book of this type the author must necessarily be dogmatic, and not all his views would be generally accepted. This reviewer finds it hard to believe that the primary pathological lesion of chronic bronchitis is " underlying vascular degeneration,' nor can he believe that any useful purpose is served by carrying out test meals as a routine in asthma. Moreover to recommend the climatic advantages of Egypt and California to the bronchitic 